INTERNATIONAL JOURNAL OF

SYSTEMATIC BACTERIOLOGY

Vol. 17, No. 2 April 1967

pp. $93-103$

Copyright 1967 by the Iowa State University Press

\title{
ANNOTATIONS FROM THE NCTC ON THE LIST OF TYPE AND REFERENCE STRAINS OF BACTERIA, SNEATH AND SKERMAN $1966^{1}$
}

S. P. Lapage, L. R. Hill, Jenifer Midgley and Jean E. Shelton

\author{
National Collection of Type Cultures, \\ Central Public Health Laboratory \\ London, N.W. 9
}

\begin{abstract}
This annotation contains a series of amendments and additions to the National Collection of Type Culture data on strains held. in the National Collection of Type Cultures which aregiven in "A list of type and reference strains of bacteria," (Sneath and Skerman 1966). Amendments or additions to the data of other Culture Collections are not included. It is suggested that copies of the list should be emended to avoid errors until a revised list is published.
\end{abstract}

\section{INTRODUCTION}

This annotation contains amendments and additions from the National Collection of Type Cultures to "A new list of type and reference strains of bacteria" published in the International Journal of Systematic Bacteriology prepared for the International Subcommittee on Numerical Taxonomy (Sneath and Skerman 1966).

In the amendments and additions the strains arearranged alphabetically in the same order and under the same nomenclature as in the list. They may be kept in the National Collection under another name, for example Chromobacterium viscosum NCTC 2416 in the list is kept in the National Collection as Corynebacterium viscosum NCTC 2416.

When a culture is requested from the National Collection of Type Cultures which has been chosen from Sneath and Skerman's list, please quote both the name and the NCTC number of the culture, and mention that it was selected from this list, in order to avoid any errors. 
The following types of amendment are given:

1. Cultures quoted with NCTC numbers which have been discarded from the National Collection of Type Cultures, e.g. "Acetobacter capsulatus NCTC 4943 discarded from NCTC."

2. Cultures maintained in the NCTC under the strain name or number quoted in the list but whose NCTC numbers were not quoted, e.g. "Aerobacter lipolyticus 'ATCC $14460^{\prime}$ kept as NCTC 10422."

3. Misquotation of NCTC numbers is corrected, e.g. "Bacillus circulans. Strain 'Ford 26' change NCTC 2601 to NCTC 2610." In case workers possess cultures of the misquoted number, the name of the organism allotted that number is given to avoid confusion. "NCTC 2601 is a discarded culture of Bacillus cereus."

4. Changes in Sneath and Skerman's annotations are prefixed by the word 'annotation.'

5. The depositor of the strain in the NCTC is given for information, where it was not quoted or where it was incorrectly attributed in the list, e.g. "Alcaligenes odorans, CCEB to NCTC."

6. Miscellaneous amendments and comments.

The amendments and additions have been made from our records and checked against other available sources in order to keep the amendments as accurate as possible.

Amendments to other Culture Collection data have not been given.

Judging by the number of amendments to the National Collection data, it would seem advisable to prepare a new list as suggested on p. 14 of Sneath and Skerman's paper, when changes and comments have been published by Culture Collections and by other workers. 


\section{ALPHABETICAL LIST OF AMENDMENTS AND ADDITIONS \\ TO SNEATH AND SKERMAN'S LIST}

Acetobacter capsulatus

Acetobacter mobilis

Acetobacter suboxydans

Ace tobacter turbidans

Achromobacter cycloclastes

Actinobacillus lignieresii

Actinomyces naeslundii

Aerobacter lipolyticus

Aeromonas shigelloides

Alcaligenes haemolysans

Alcaligenes odorans

Arthrobacter simplex

Arthrobacter tumescens

Arthrobacter ureafaciens

Bacillus alvei

Bacillus aminovorans

Bacillus anthracis

Bacillus badius

Bacillus brevis

Bacillus cereus
NCTC 4943 discarded from NCTC

NCTC 6428 discarded from NCTC

NCTC 3734 discarded from NCTC

NCTC 6249 discarded from NCTC

NCTC 2572 discarded from NCTC

NCTC 4976 = strain "CM 2".

Annotation: P. Martin to NCTC

Strain "279" kept as NCTC 10301. Annotation: Pine to NCTC

"ATCC 14460" kept as NCTC 10422. Annotation: ATCC to NCTC

Strain "M51" kept as NCTC 10360. Annotation: ATCC to NCTC

NCTC 5043 discarded from NCTC

Strain "14-45", "CCEB 554" kept as NCTC 10416.

Annotation: CCEB to NCTC

NCTC 4215 discarded from NCTC

NCTC 4216 discarded from NCTC

NCTC 7811 discarded from NCTC

Strain "Lochhead" kept as NCTC 6352 Annotation: Gibson to NCTC. Quoted as "Lochhead 127", Smith et al., 1964 , J.gen.Microbio1., $34,269 \overline{-2} 7 \overline{2}$

NCTC 2870 discarded from NCTC

Strain "Vollum" kept as NCTC 10340.

Annotation: Gibson to NCTC

Strain "N.R. Smith 663" kept as NCTC 10333.

Annotation: Gibson to NCTC

NCTC 2611. Annotation: Gibson to NCTC

Strain "Gibson 971" kept as NCTC 2599. Annotation: Gibson to NCTC 
Bacillus circulans

Bacillus coagulans

Bacillus firmus

Bacillus laterosporus

Bacillus lentus

Bacillus licheniformis

Bacillus licheniformis

Bacillus macerans

Bacillus megaterium

Bacillus pantothenticus

Bacillus polymyxa

Bacillus pumilus

Bacillus sphaericus

Bacillus stearothermophilus

Bacillus subtilis
Strain "Ford 26", change NCTC 2601 to NCTC 2610.

Annotation: Gibson to NCTC.

(NCTC 2601 is a discarded culture of Bacillus cereus)

Strain "N.R. Smith 609" kept as NCTC 10334.

Annotation: Gibson to NCTC

Strain "N.R. Smith 613" kept as NCTC 10335.

Annotation: Gibson to NCTC

Strain "Ford 6" kept as NCTC 6357. Annotation: Gibson to NCTC.

Comment on annotation: Smith et al., 1964 , J.gen.Microbiol., $34,269-272$, propose ATCC 10840 as type culture. ATCC $10840=$ "Gibson 165" kept as NCTC 4824. Gibson to NCTC.

NCTC 2586 discarded from NCTC

Strain "Gibson 46" kept as NCTC 10341. Annotation: Gibson to NCTC

Strain "N.R. Smith 888 " kept as NCTC 6355.

Annotation: Gibson to NCTC

Strain "Ford 19" kept as NCTC 10342. Annotation: Gibson to NCTC

NCTC 8162. Change strain "CN 3208" to "CN 3028", Smith et al., 1964. J.gen. Microbiol., $3 \overline{4}, \frac{26}{26} 9-272$ and NCTC records. Annotation: Proom to NCTC

"ATCC 842" kept as NCTC 10343. Annotation: Gibson to NCTC

Strain "Král" kept as NCTC 10337. Annotation: Gibson to NCTC

Strain "Gibson 1013" kept as NCTC 10338.

Annotation: Gibson to NCTC

Strain "N.R. Smith T18", "NCA 26", kept as NCTC 10339.

Annotation: Gibson to NCTC

NCTC 3610. Change strain "Smith 704" to "N.R. Smith 744", NCTC records 
Bacterionema matruchotii

Bordete1la pertussis

Brevibacterium ammoniagenes

Bruce1la abortus

Brucella melitensis

Bruce1la me1itensis

Bruce11a suis

Chromobacterium violaceum

Chromobacterium viscosum

Clostridium botulinum

Clostridium chauvoei

Clostridium novyi

Clostridium perfringens

Clostridium septicum

Clostridium tertium

Corynebacterium bovis

Corynebacterium pseudotuberculosis
"ATCC 14266" kept as NCTC 10254. Annotation: Gilmour to NCTC

NCTC 9797. Annotation: Change "Pittman to ATCC to NCTC" to"Kendrick to Standfast to NCTC"

NCTC 2398 discarded from NCTC

Strain "544" kept as NCTC 10093. Annotation: Payne to NCTC

NCTC 7470. Annotation: Cruickshank to NCTC

Strain "16M" kept as NCTC 10094. Annotation: Payne to NCTC

Strain "1330" kept as NCTC 10316. Annotation: Brinley-Morgan to NCTC

NCTC 9757. Annotation: Sneath to NCTC

NCTC 2416.

Annotation: Grimes to NCTC

NCTC 7272. Annotation: Change "PHLS to NCTC" to"Microbiological Research Establishment, Porton to B.C. Hobbs to NCTC"

NCTC 8070. Annotation: Gaiger to Henderson to NCTC

Change NCTC 541 to NCTC 538 quoted Willis and Hobbs, 1959. NCTC $538=$ Strain "Jolly". Annotation: Weinberg to Robertson to NCTC. (NCTC 541 is a culture of Clostridium tertium)

NCTC 8237. Annotation: Burroughs Wellcome to NCTC

NCTC 547 change strain "Pasteur 111" to"Pasteur III"

NCTC 541. Change"PII Co1" to'PII Col 54".

Annotation: Petrie to Robertson to NCTC

ATCC 7715. Strain "Evans" kept as NCTC 3224. Annotation: Evans to NCTC

NCTC.3450, Annotation: Buxton to Petrie to NCTC 
Corynebacterium rubrum

Escherichia coli

Escherichia coli

Escherichia freundii

Flavobacterium meningosepticum

Haemophilus aegyptius

Haemophilus haemoglobinophilus

Haemophilus haemolyticus

Haemophilus vaginalis

Klebsie11a edwardsii

Klebsie11a edwardsii

Xlebsie11a pneumoniae

Klebsiella rhinoscleromatis

Kurthia zopfii

Lactobacillus 1eichmannii

Leptotrichia buccalis

Leptotrichia dentium

Listeria monocytogenes

Listeria monocytogenes
"ATCC 14898" kept as NCTC 10391. Annotation: ATCC to NCTC

NCTC 9001. Strain "U5/41".

Annotation: Kauffmann to NCTC

NCTC 86. Annotation: Lister Institute to NCTC

NCTC 9750. Annotation: ATCC to NCTC

NCTC 10016. Annotation: King to NCTC

NCTC 8502. Annotation: pittman to NCTC

Strain "X111" change to "XIII". Annotation: Friedberger to Kristensen to Fildes to $\mathrm{NCTO}$

NCTC 8479. Annotation: Pittman to NCTC

Strain "Dukes' 594" kept as NCTC

10287. Annotation: Zinnemann to NCTC

NCTC 5054. Annotation: Snijders to NCTC. Add variety edwardsii

NCTC 9496. Annotation: Edwards to NCTC

NCTC 9633. Annotation: Edwards to NCTC

NCTC 5046. Annotation: Snijders to NCTC

NCTC 404. Annotation: Rettger to NCTC. Change ATCC 405 to NCTC 405.

NCTC 7854 discarded from NCTC

ATCC 14201. Add NCTC 10249

NCTC 10206 add strain "LDM 3"

NCTC 10357. Delete strain "58-xxiii" add strain "53 XXIII".

Annotation: Murray to NCTC. From a rabbit.

NCTC 7973 add strain "58 XXIII". Annotation: Webb to NCTC. From a guinea pig. 
Listeria monocytogenes (cont.) Annotation: Both strains from laboratory epidemic described in Murray, Webb \& Swann, 1926. J.Path.Bact., $\underline{29}, 407$.

Micrococcus agilis

NCTC 2676. Annotation: Piorkowski to Pribram Collection to Breed to ATCC to NCTC

Micrococcus hyicus

NCTC 10350. Annotation: ATCC to NCTC

Micrococcus luteus

Micrococcus rhodochrous

$\underline{\text { Micrococcus roseus }}$

NCTC 8512. Annotation: ATCC to NCTC

Strain "KMRh" kept as NCTC 10210. Annotation: Gordon to NCTC

NCTC 7523. Annotation: Shaw et al., 1951 propose NCTC 7520 as type species. Hi11, 1959 suggested NCTC 7523

Moraxe11a 1acunata

NCTC 7784. Comment: We classify this strain as Moraxella non-liquefaciens in NCTC. In our hands it fails to 1iquefy serum or gelatin, see Bövre \& Henriksen, Acta path.microbiol. scand., 1962, 56, 223-228 and Bövre, 1964, ibid., $\underline{61}, 457-473$.

Moraxe1la 1woffii

NCTC 5866. Add strain "Lwoff"

Mycobacterium acapulcensis

NCTC 10270. Annotation: Bojalil to NCTC

Mycobacterium balnei

Strain "X" kept as NCTC 10011. Annotation: ATCC to NCTC

Mycobacterium flave'scens

Mycobacterium fortuitum

Mycobacterium gordonae

Mycobacterium kansasii

NCTC 10271. Annotation: Bojalil to NCTC

Strain "IOC-1" probably J.C. Cruz 1, if so kept as NCTC 10394.

Annotation: Gordon to NCTC

NCTC 10267. Annotation: Bojalil to NCTC

Annotation: Strain "P22" kept as NCTC $10268=$ ATCC 14471. Bojalil to NCTC

Mycobacterium marinum

ATCC 927 = strain "Aronson", kept as NCTC 2275.

Annotation: Aronson to NCTC

Mycobacterium peregrinum

NCTC 10264. Annotation: Bojalil to NCTC 
Mycobacterium rubrum

Mycobacterium runyonii

Mycobacterium smegmatis

Mycobacterium tuberculosis

Mycobacterium ulcerans

Mycoplasma mycoides

Neisseria caviae

$\underline{\text { Neisseria flavescens }}$

Neisseria gonorrhoeae

Neisseria haemolysans

Neisseria meningitidis

Neisseria ovis

Nocardia farcinica

Nocardia salivae

Paracolobactrum arizonae

$\underline{\text { Pasteure1la multocida }}$

Pasteure11a pestis

Pediococcus cerevisiae

Propionibacterium arabinosum

Propionibacterium jensenii

Propionibacterium peterssonii
NCTC 1468 discarded from NCTC

NCTC 10269. Annotation: Bojalil to NCTC

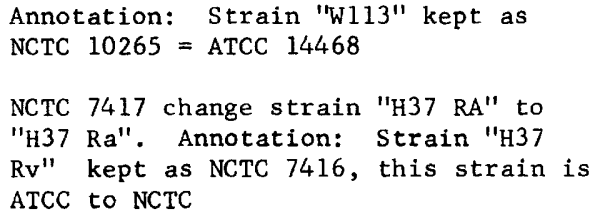

NCTC 7816 discarded from NCTC redeposited as NCTC 10417. Annotation: NCTC 10417 Buckle to NCTC

NCTC 3278 discarded from NCTC

Strain "GP 11" kept as NCTC 10293. Annotation: Pelczar to NCTC

NCTC 8263.

Annotation: Branham to NCTC

NCTC 8375. Annotation: Wilson to NCTC

NCTC 5414 discarded from NCTC

Strain 'M 1027" kept as NCTC 10025. Annotation: Branham to NCTC

Was not deposited with the NCTC from our records.

NCTC 4524. Annotation: ATCC to NCTC

NCTC 10207. Add strain "B61"

NCTC 8297. Add strain "DC 5". Annotation: Edwards to NCTC

NCTC 3195. Annotation: Change

"Schutz" to"Schütze"

NCTC 5923. Annotation: Change

"Schutz" to "Schütze"

NCTC 8066. Annotation: Pederson to NCTC

NCTC 5958 discarded from NCTC

NCTC 5960 discarded from NCTC

NCTC 5962 discarded from NCTC 
Propionibacterium technicum

Protaminobacter alboflavus

Protaminobacter ruber

Proteus morganii

Proteus vulgaris

Providencia alcalifaciens

Pseudomonas aeruginosa

$\underline{\text { Pseudomonas aeruginosa }}$

Pseudomonas alcaligenes

Pseudomonas barkeri

Pseudomonas chlororaphis

Pseudomonas indoloxidans

Pseudomonas $\underline{\text { iodinum }}$

Pseudomonas maltophilia

Pseudomonas mucidolens

Pseudomonas pseudomallei

Rhodospirillum rubrum

Salmone1la choleraesuis

Salmone11a typhi
NCTC 5965 discarded from NCTC

NCTC 2875 discarded from NCTC

NCTC 2879 discarded from NCTC

NCTC 235. Annotation: Morgan to Lister Institute to NCTC

NCTC 4175. Add strain "Lehmann". Annotation: Lehmann to Pribram Collection to NCTC

"ATCC 9886" kept as NCTC 10286

NCTC 6750. Annotation: Schütze to NCTC

Strain "RH 815" kept as NCTC 10332. Annotation: Lysenko to NCTC

"ATCC 14909" kept as NCTC 10367.

Add strain "Hugh 1577".

Annotation: ATCC to NCTC

NCTC 393 discarded from NCTC

ATCC 9446. Delete NCTC 9392, ATCC

9446 is not kept in the NCTC. (NCTC 9392 is a strain of Neisseria meningitidis)

NCTC 2760 discarded from NCTC

NCTC 9742. Annotation: ATCC to NCTC. Comment: We have no record of "Davis" as strain name

NCTC 10257. Annotation: ATCC to NCTC

ATCC 4685 kept as NCTC 8068.

Annotation: ATCC to NCTC

NCTC 4845 strain "S $397 "=$ ATCC

15682. Annotation: W.A. Young to NCTC

NCTC 7762 discarded from NCTC

NCTC 5735. Add strain "1348, K34". Annotation: Kauffmann to NCTC

NCTC 8385. Add strain "Ty2". Annotation: Felix to NCTC 
Salmonella typhimurium

Serratia marcescens

Serratia marcescens

Shige1la dysenteriae

Spirillum mancuniense

Staphylococcus afermentans

Staphylococcus aureus

Staphylococcus lactis

Staphylococcus saprophyticus

Streptobacillus moniliformis

Streptococcus agalactiae

Streptococcus asalignus

$\underline{\text { Streptococcus faecalis }}$

Streptococcus faecium

Streptococcus lactis

Streptococcus uberis

Vibrio comma

Vibrio cuneatus

Vibrio cyclosites

Vibrio fetus
NCTC 74. Add strain "Mutton". Annotation: Lister Institute to NCTC

NCTC 1377. Annotation: Kluyver to NCTC

NCTC 10211. Add strain "BS 303". Annotation: Kocur to NCTC

NCTC 4837. Annotation: Scott to NCTC

NCTC 9582 discarded from NCTC

NCTC 2665. Annotation: Fleming to NCTC

NCTC 8532. NCTC 8532 is designated as the neotype. Opinion 17, Jud. Comm. Annotation: Cowan to NCTC

NCTC 7564. Annotation: Gibson to NCTC

Strain "S41". Change NCTC 7297 to NCTC 7292. (NCTC 7297 is a strain of Salmone1la arizonae).

Annotation: Cowan to NCTC

NCTC 8069. Klienebergex-Nobel to NCTC

NCTC 8181, Annotation: Stableforth to NCTC

NCTC 2294 discarded from NCTC

NCTC 775. Add strain "Tissier" Annotation: Pasteur Institute to Brown to NCTC in 1920. Redeposited Hannay to NCTC in 1950.

NCTC 7171. Annotation: Grumbach to NCTC

NCTC 6681. Add strain "OJ"

NCTC 3858. Add strain "Diernhofer"

NCTC 8021. Annotation: Standards

Laboratory, Colindale to NCTC

NCTC 2583 discarded from NCTC

NCTC 2581 discarded from NCTC

NCTC 10354 is strain "X/161/5".

Annotation: Park to NCTC.

Comment: Strain "Milton" is NCTC 5850. Dalling to NCTC 
Vibrio neocistes

Vibrio percolans

Xanthomonas proteamaculans
NCTC 2582 discarded from NCTC

NCTC 1937. Add strain "Mudd". Annotation: Mudd to NCTC

NCTC 394 discarded from NCTC

\section{REFERENCE}

Sneath, P.H.A. and Skerman, V.B.D. (1966). A list of type and reference strains of bacteria. Int.J.Syst.Bact., 16: 1-133. 